\title{
Possible roles for stanniocalcin during early skeletal patterning and joint formation in the mouse
}

\author{
S E Stasko and G F Wagner \\ Department of Physiology, Faculty of Medicine and Dentistry, University of Western Ontario, London, Ontario, Canada, N6A 5C1 \\ (Requests for offprints should be addressed to G F Wagner; Email: graham.wagner@med.uwo.ca)
}

\begin{abstract}
Stanniocalcin (STC) is a polypeptide hormone discovered first in fish and more recently in mammals. In mammals, the gene is widely expressed and the hormone is, so far, known to be involved in regulating the transport of calcium or phosphate across renal and gut epithelia, and into neuronal cells. Gene expression is also high during development, and in an earlier study we mapped the temporal and spatial pattern of gene expression in the mouse urogenital system. Our data suggested that STC probably acted as a signaling molecule that was produced in mesenchyme cells and targeted to epithelial cell layers in both kidney and testes. Here we have examined STC mRNA and protein distributions between developmental stages E10.5 and E18.5 in the axial and appendicular skeleton. In the axial skeleton, STC was transiently expressed in a rostral-caudal fashion during vertebral development; protein appeared to be made in intervertebral disc mesenchyme cells and targeted to vertebral hypertrophic and prehypertrophic chondrocytes. By stage E18.5, the STC gene was active only in vertebral
\end{abstract}

perichondrocytes. The pattern of expression in the appendicular skeleton was equally striking. Early in development, STC gene expression defined the initial lengths of bone primordia. The gene was expressed in mesenchyme cells at either ends of precartilaginous condensations defining future long bones and the secreted protein was targeted to the chondroblasts. Later on during joint formation, STC was highly expressed in interzone cells that defined all future joints. After cavitation, STC gene expression was greatest in perichondrocytes lining the joints. Underlying resting, proliferative and prehypertrophic chondrocytes appeared to be the targets of STC both during and after cavitation. Therefore, its pattern of expression was indicative of a role in early skeletal patterning and joint formation. Moreover, as occurs during urogenital development, it appeared that STC is made in undifferentiated mesenchyme cells and sequestered by those destined to differentiate.

Journal of Endocrinology (2001) 171, 237-248

\section{Introduction}

Stanniocalcin (STC) is a $50 \mathrm{kDa}$ disulfide-linked, homodimeric glycoprotein that was discovered first in fish and more recently in mammals. In mammals and fish the STC gene is widely expressed and in both groups the hormone plays a part in mineral homeostasis (Wagner 1994, Wagner et al. 1986, Chang et al. 1995, 1996, Olsen et al. 1996, McCudden et al. 2001). In mammals, STC promotes the reabsorption of inorganic phosphate across renal and intestinal epithelia, and reduces intestinal calcium transport (Wagner et al. 1997, Madsen et al. 1998, Varghese et al. 2000). STC also has a role in neuronal cell differentiation and, by increasing the rate of phosphate uptake, may protect neurons from ischemic insults (Zhang et al. 1998, 2000).

STC is also highly expressed during embryogenesis, which is indicative of it having some importance to development (Varghese et al. 1998). This has prompted us and others to analyze in more detail the temporal and spatial patterns of expression of STC during development, using mouse as a model. The first of these studies traced the patterns of STC immunoreactivity (STCir) in axial skeletal development up to stage E14.5, and revealed high levels in cells of the chondrocyte lineage, particularly in intervertebral discs (Jiang et al. 2000). We then examined mouse urogenital development between stages E10.5 and 18.5 and found intriguing patterns of gene expression in kidney and testes: in both organs the gene was expressed in mesenchymal cells, whereas the protein was much more evident in adjacent epithelial cells (Stasko \& Wagner 2001). Findings such as these were suggestive of local STC signaling between the two cell types. In a follow-up study we examined patterns of gene expression in the uterus after blastocyst implantation. Here we observed that STC was expressed uniquely in mesometrial stromal cells and targeted to/sequestered by decidualizing anti-mesometrial cells, indicative of a role in decidualization and uterine remodeling (Stasko et al. 2001). Hence, these latter studies demonstrated the 
necessity of screening for both mRNA and protein to obtain some indication as to the source of the hormone and the direction in which it appeared to signal. The importance of STC to development has been further revealed through transgenesis, as the over-expression of human (h) $S T C$ in the mouse leads to significant reductions in birth weight, adult dwarfism and significant increases in serum phosphate concentrations (Varghese et al. 2000). The present study attempted to build on this previous body of work by delineating the patterns of distribution of STC mRNA and protein during all relevant stages of axial and appendicular skeletal development (E10.5 to E18.5). The data suggests that, as in other tissues, STC signals locally between adjacent cell types and appears to be involved in both skeletal patterning and joint formation.

\section{Materials and Methods}

\section{Tissue preparation}

Standardized timed mating schemes were arranged between 6-week-old CD-1 mice. Embryos were isolated between days E10.5 and E18.5, fixed overnight at $4{ }^{\circ} \mathrm{C}$ in $4 \%$ paraformaldehyde in PBS ( $\mathrm{pH} 7 \cdot 5)$, dehydrated and embedded in paraffin, and sectioned at $6 \mu \mathrm{m}$ for immunocytochemistry and in situ hybridization. Ten to 16 fetuses were evaluated per stage from multiple pregnancies.

\section{Immunocytochemistry}

The antiserum used was generated in rabbits against baculovirus-expressed recombinant hSTC and has been extensively characterized by immunocytochemistry (ICC), western blot analysis and radioimmunoassay for specificity in humans and rodents (Varghese et al. 1998, Niu et al. 2000). For ICC staining, slides were deparaffinized and rehydrated, and endogenous peroxidase activity was quenched by a $30 \mathrm{~min}$ treatment in $0 \cdot 3 \%$ hydrogen peroxide in methanol. The staining procedure involved pretreatment of tissue sections with $10 \%$ normal goat serum in diluent buffer (PBS) to reduce non-specific staining, followed by an overnight incubation at $4{ }^{\circ} \mathrm{C}$ with a 1:1000 dilution of the antiserum. Sites of antibody binding were then visualized with the vectastain avidinbiotin complex peroxidase detection system according to the manufacturer's instructions (Vector Labs, Burlingame, CA, USA). Slides were counterstained with Harris hematoxylin, dehydrated and mounted. Control procedures involved the application of antiserum preabsorbed with hSTC $(1 \mathrm{mg} / \mathrm{ml})$ in lieu of antiserum alone.

\section{In situ hybridization}

In situ hybridization (ISH) was carried out using a previously established procedure with an $815 \mathrm{bp}$ cDNA encoding 247 amino acids of mouse STC for generating sense and antisense riboprobes (Varghese et al. 1998, Wong et al. 1998). The plasmid was linearized with restriction enzymes for the synthesis of sense and antisense cRNAs to a specific activity of $1-3 \times 10^{8}$ c.p.m./mg of RNA with $\left[{ }^{35} \mathrm{~S}\right] \mathrm{UTP}$ according to the manufacturer's instructions (Pharmacia Biotech, Baie d'Urfe, QC, Canada). Briefly, hydrated tissue sections were treated with proteinase $\mathrm{K}$, post-fixed in $4 \%$ paraformaldehyde, acetylated, dried and then hybridized overnight at $50{ }^{\circ} \mathrm{C}$ in a solution of $50 \%$ formamide, $0.4 \mathrm{M} \mathrm{NaCl}, 10 \mathrm{mM}$ Tris $\mathrm{HCl} \mathrm{pH} 8 \cdot 0,1 \mathrm{mM}$ EDTA $\mathrm{pH} 8 \cdot 0,1 \times$ Denhardt's solution, $10 \%$ dextran sulfate, $20 \mathrm{mM}$ dithiothreitol, $600 \mu \mathrm{g} / \mathrm{ml}$ denatured yeast transfer RNA, and $0 \cdot 1 \mu \mathrm{g}$ labeled RNA probe/ml solution. After extensive posthybridization washes, single-stranded riboprobe was digested with RNase A. Slides were finally dehydrated, air-dried, dipped in Kodak NBT2 nuclear track emulsion and then stored at $4{ }^{\circ} \mathrm{C}$ in light-tight boxes containing silica gel as desiccant for 13-21 days. Slides were developed in Kodak D-19, counterstained with hematoxylin, dehydrated and mounted under coverslips. Digital images were captured using a SPOT camera (Diagnostic Instruments, Sterling Heights, MI, USA).

\section{Results}

\section{$S T C m R N A$ and protein in axial skeleton}

In axial skeleton, $S T C$ gene expression proceeded in a rostral-caudal direction, coincident with the progressive differentiation, segmentation and patterning of sclerotomal somites into components of the axial skeleton, beginning at developmental stage E10.5. By stage E11.5, gene expression became restricted to the rostral, less condensed halves of a dozen or more individual sclerotomal segments along the post-cranial axis (Fig. 1A). Little or no signal was found in the caudal half, characterized by its greater cell density (Fig. 1B). In contrast, STC protein was readily apparent in cells of both halves of the somite (Fig. 1C).

With the successive differentiation of these sclerotomal components over developmental stages E12.5-E13.5 into vertebrae, ribs and intervertebral discs, an even more restricted pattern of STC gene expression was observed. The highest levels of STC mRNA were now in intervertebral disc anlagens as they developed in a rostral to caudal direction. A good example of this is illustrated in Fig. 1D and $\mathrm{E}$, where the most intense hybridization was found in condensing lumbar-sacral intervertebral discs between the precartilage vertebral bodies. In contrast, gene expression was undetectable in the rostral, more developmentally advanced thoracic vertebrae and ribs, now defined by a perichondrium. Gene expression was also absent from the more caudal and less developmentally advanced coccygeal somites. 
STC protein was found in stage-specific patterns of staining between stages E12.5 and E13.5. In the lumbar-sacral region, STC protein co-localized in areas of high gene expression in the condensing intervertebral discs (Fig. 1F) and in primitive vertebrae also (Fig. 1F,G). By stage E13.5, STC mRNA levels had decreased in the developmentally more advanced cervical-thoracic regions, yet they still contained high levels of STCir. This was particularly evident in the newly formed vertebral and rib perichondria, intervening fibrocartilage and select chondrocytes (Fig. 1D inset, arrowheads). By stage E14.5, however, STC gene activity had moved caudally and was now greatest in the intercoccygeal discs, whereas all discs rostral to the tail had little if any STC mRNA (not shown). Intervertebral discs again contained the most STCir, although low levels were found in adjacent regions of lower cell density destined to become coccygeal vertebrae (not shown). STC protein was also now evident in the chondrocytes of future skeletal elements, once they had been defined by a perichondrium (not shown).

With the completion of tail somite differentiation at stage E15.5 and commencing maturation of cartilaginous elements, the entire axial skeleton now exhibited only low levels of STC expression in intervertebral disc mesenchyme cells. Instead, gene expression was now confined to the perichondrium of vertebral elements (Fig. 1H). STC protein co-localized with mRNA in perichondrial cells and was also found in the chondrocytes of developing skeletal elements (Fig. 1I,J). Figure 1J highlights the heterogeneous nature of STCir in differentiating chondrocytes: those containing high levels of STCir were more likely to have less compact chromatin, indicative of greater cellular activity. Over the remaining days of embryogenesis there was a marked reduction in STC synthesis, coinciding with further axial skeleton development.

Overall, our protein localization data in axial skeleton agreed with earlier findings (Jiang et al. 2000). However, with the added gene expression data our findings further revealed that, early on, STC appears to be produced by one cell type and targeted to adjacent cells in condensing perichordal mesenchyme, possibly abetting in early segmentation/patterning events. Later on, however, STC is involved in intervertebral disc formation and chondrogenesis in skeletal elements. A diagrammatic summary of these events is shown in Fig. 2.

\section{STC in appendicular skeleton}

Activation of STC gene transcription coincided with three major events in limb development: condensation, growth and segmentation. Low levels of STC mRNA and protein were first seen in E9.5 limb buds (data not shown). However by developmental stage E11.5, mRNA and protein were both clearly evident in cells bordering the proximal and distal regions of precartilaginous condensations within the limbs (Fig. 3A). These rod-shaped digital primordia were visible in the hind limb under bright field illumination (Fig. 3B). Low levels of STC mRNA were also found in mesenchyme cells surrounding the developing digital rays (Fig. 3A, arrowheads). Most of the STCir was confined to mesenchyme cells immediately around the developing digits (Fig. 3C). A similar pattern was seen in E12.5 fore limbs (data not shown).

By stage E13.5, in the developmentally more advanced fore limb (approximately half a day ahead of the hind limb) the highest levels of STC gene expression were now confined to the mesenchymal condensations at the most distal ends of the developing metacarpal elements (Fig. 3D,E, arrowheads), coinciding with the development of the presumed metacarpal-phalangeal joint. A moderate level of gene expression was also found between more proximal carpal elements. In contrast, chondroblasts in these carpal and metacarpal elements were generally devoid of STC mRNA, but in some cases were positively stained for STC protein (Fig. 3F, arrows). Regions containing the highest levels of STC mRNA contained little or no protein (Fig. 3F, arrowhead).

Subsequently, between developmental stages E14.5 and $18 \cdot 5$, STC gene expression became concentrated in all regions of joint formation (or future joint formation) in fore and hind limb digits, and in shoulder/elbow/wrist and hip/knee/ankle regions. In contrast, STC protein was evident in a large proportion of chondrocytes within the respective cartilage elements, and in some perichondrocytes. A good example of this is the E15.5 hind limb shown in Fig. 3G-I, where STC mRNA is present in almost all regions of bone apposition. Here, it can be seen that the greatest levels of expression were found in the interzones of metatarsal-phalangeal joints (Fig. 3G-I, large red arrowheads) and to a lesser extent in more distal interphalangeal joint regions (Fig. 3G-I, small red arrowhead). Moderate signal was also evident in cells lining the spaces between the various tarsal elements (e.g. cuneiformes, naviculare, cuboideum), but cells lining the talus-calcaneus joint had a particularly high level of expression (Fig. 3G, yellow arrowhead). However, as noted above, Fig. 3J reveals that the pattern of STC protein staining was markedly different. Chondrocytes of the tarsal and digit cartilaginous elements contained the highest levels of STCir, whereas lower levels were seen in gene-expressing cells of the perichondrium and actual joints regions (Fig. 3J). Skin was also highly immunoreactive for STC (Fig. 3J). The pattern was similar in E16.5 hind limb, except for the fact that STC gene activity was now also evident in perichondrocytes extending back from the joint interzones on the lateral margins of cartilage elements (Fig. 3K, arrowheads). Once again, however, most of the STCir was in chondrocytes, not the perichondrium. Figure $3 \mathrm{~L}$ is a high magnification of the hind limb tarsal elements shown in Fig. 3K. Highlighted here are the high levels of STCir in chondrocytes that apparently do not produce STC (arrow), as compared with the weak 
STCir in cells of the interzone (iz) and perichondrium (arrowhead) that expressed the gene at high levels.

Other more proximal appendicular regions exhibiting strong gene expression at developmental stage E15.5 included the elbow and knee joints (Fig. 3M), where the highest levels coincided with cavitation (arrows). After cavitation, STC gene expression became confined to the joint perichondrial surfaces (see Fig. 4C for an example of this). High levels of STC mRNA were also apparent in future articular surfaces of the humerous-scapula (or gleno-humeral) shoulder joint (Fig. 4A, and inset, arrowhead), whereas STC protein was evident in both perichondrocytes and chondrocytes of the underlying hyaline cartilage (Fig. 4B).

By E18·5, STC gene expression was highest following cavitation of the most distal inter-phalangeal joints of the fore limb and STC mRNA was again restricted to the outermost layer of cells forming the opposing articular surfaces (Fig. 4C,D, arrowheads and arrows). STC protein co-localized with the mRNA, but was also evident in the underlying chondrocytes and associated ligaments (Fig. 4E, arrows). Similar finding were observed in the hind limbs, particularly along the edges of the naviculare skeletal element of the ankle (data not shown).

The distribution of STC MRNA and protein in the epiphyseal growth plate of an E17.5 femur is illustrated at low magnification in Fig. 4, panels $\mathrm{F}-\mathrm{H}$. As in the case of smaller long bones, STC gene expression in the E17.5 femur was restricted to the thin layer of perichondrocytes defining the articular surfaces (Fig. 4 I-1, I-2, arrows). In contrast, high levels of STCir were also found in chondrocytes of the resting, proliferative and hypertrophic zones of the growth plate (Fig. $4 \mathrm{I}-3, \mathrm{~J}-\mathrm{M}$ ). Cells of the prehypertrophic and hypertrophic zones appeared to contain the least amount of STC protein (Fig. 4J, K), whereas the highest levels of STCir were found in osteoblasts of newly forming trabecular bone (Fig. 4L). In all of the above studies, the use of antiserum preabsorbed with hSTC (for ICC) and radiolabeled sense probes (for ISH) produced no specific staining or hybridization respectively (data not shown)

In summary, STC mRNA in appendicular skeleton was restricted to a discrete population of cells in regions before (regional mesenchymal condensation), undergoing (interzone) or completing (articular surface) joint formation. In contrast, the protein was most abundant in interdigital mesenchyme and chondrocytes. This clear-cut discrepancy between mRNA and protein is indicative of a signaling pathway whereby STC is made in interzone cells and perichondrocytes and targeted to and accumulates in chondrocytes in the process of differentiation.

\section{Discussion}

In an earlier study, we characterized the patterns of STC gene expression and protein distribution in developing mouse kidney and testis. In both organs, a mesenchyme-epithelial signaling pathway was evident, whereby STC was produced in mesenchyme cells and targeted to/sequestered by nearby epithelial cells (Stasko \& Wagner 2001). The present study has now revealed equally distinctive patterns of STC mRNA and protein localization during skeletogenesis, coinciding with initial patterning and subsequent differentiation of the axial and appendicular skeletons. Furthermore, it appears that, as in the urogenital system, the gene is expressed for the most part in undifferentiated cells and targeted to cartilage cells in various stages of terminal differentiation. Because STCir was clearly evident in cells having no apparent gene activity, we are assuming, as we have in previous studies, that these cells are targets of STC and have sequestered the hormone (Deol et al. 2000, Stasko \& Wagner 2001, Stasko et al 2001). We are further assuming that the STC they contain is derived from nearby mesenchymal cells, perichondrocytes, or both. Nonetheless, without evidence to the contrary, we still cannot entirely rule out the alternative explanation that there are indeed low levels of STC mRNA in differentiating chondrocytes that escaped detection by our isotopic in situ technique.

\section{STC in the axial skeleton}

The pattern of STC gene expression in axial skeleton, as summarized in Fig. 2, was most like that reported for the transforming growth factor $\beta 3$ (TGF $\beta 3$ ) gene. TGF $\beta 3$ mRNA is found early in condensations of perichordal mesenchyme between vertebral bodies, then the discs, and later the perichondrium (Pelton et al. 1990, Millan et al. 1991, Schmid et al. 1991). Other TGF $\beta 3$ superfamily members with overlapping patterns of expression included activins and inhibins. Like STC, mRNAs encoding both $\beta A$ and $\beta B$ subunits are found in developing intervertebral discs, along with follistatin, an activin binding protein (Feijen et al. 1994). The mouse XlHbox gene is also expressed in segmented mesoderm along the anterior-posterior axis and in intervertebral discs (Deutsch et al. 1988, Oliver et al. 1988, Wallin et al. 1994). Pax1 is expressed in perichordal mesenchyme and later in discs, where it shows temporal and spatial similarities with STC, albeit spanning a longer period of time (Deutsch et al. 1988, Wallin et al. 1994). Interestingly, spontaneous Pax1 mutants almost always lack vertebral bodies and intervertebral discs (Wallin et al. 1994).

The patterns of expression of STC mRNA and protein are indicative of a role for STC in axial patterning and growth of the intervertebral discs, vertebrae and ribs. The notion that STC regulates these events is also supported by recent data showing that the over-expression of STC in mice reduces growth, pre- and post-natally (Varghese et al. 2000), the implication being that skeletal maturation has 


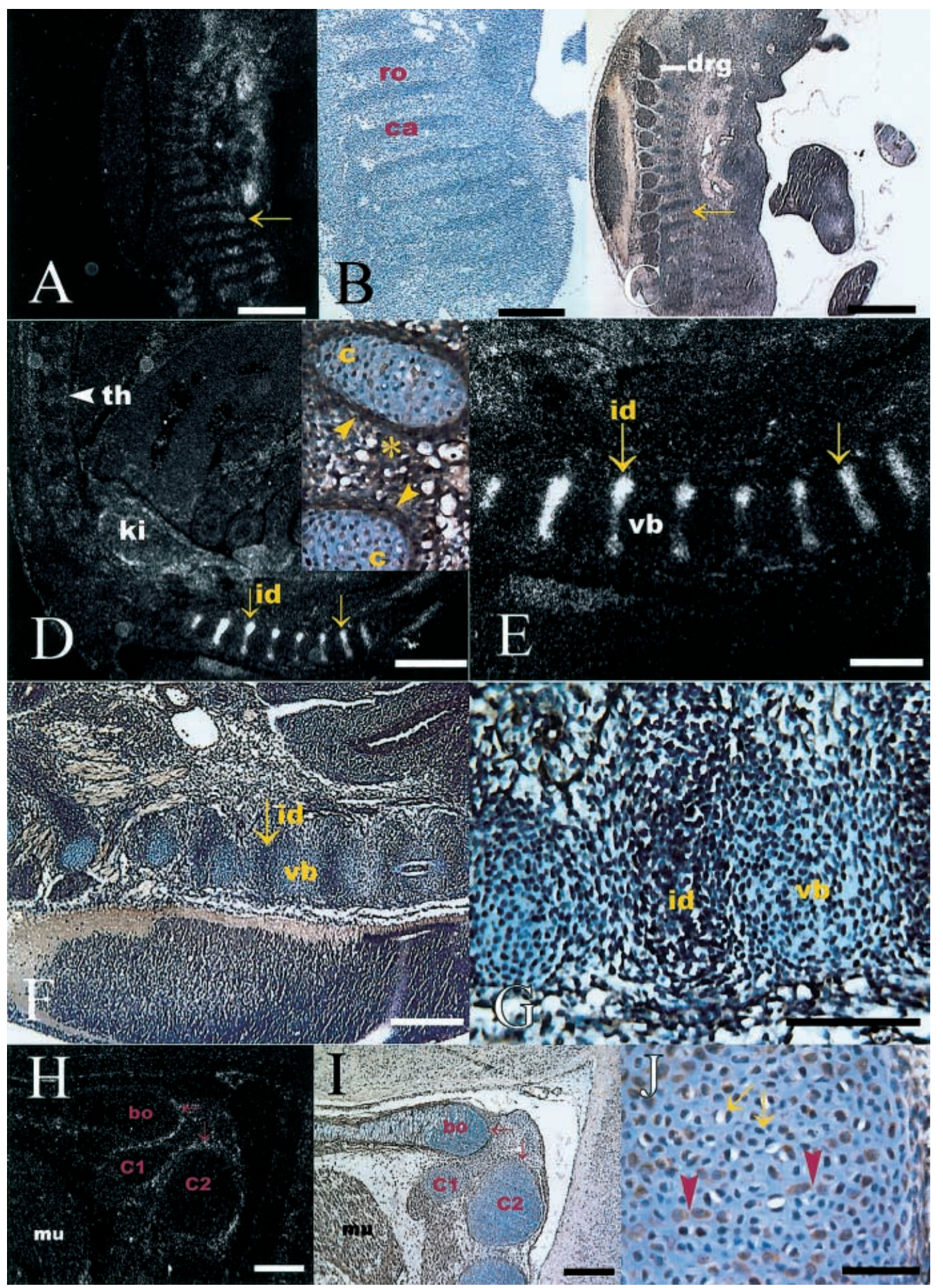

Figure 1 (see legend on page 245) 


\section{Axial}

E11.5

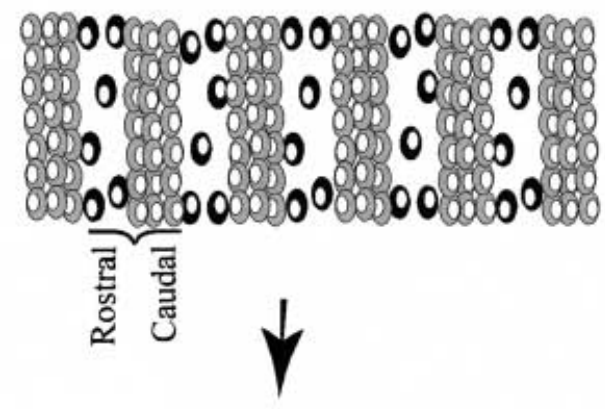

E14.5

\section{Appendicular}

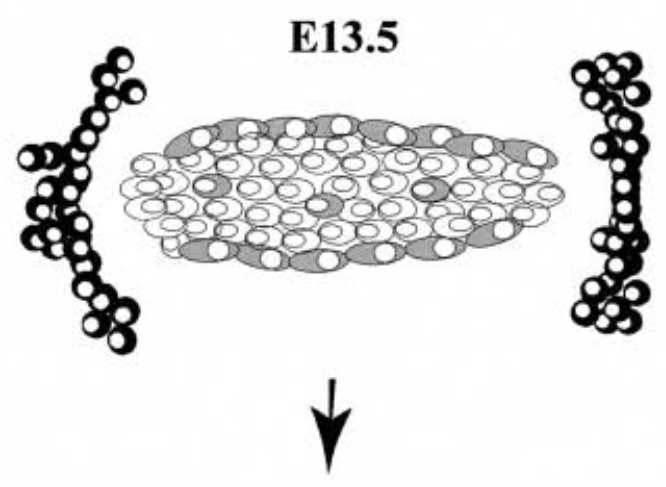

E14.5

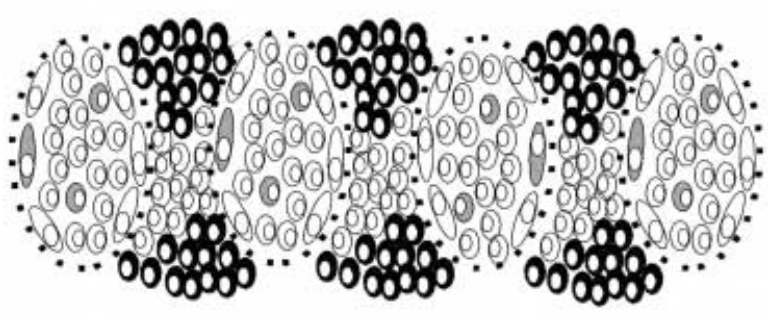

1

E18.5

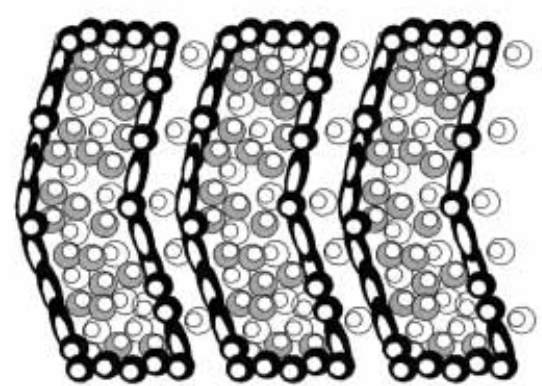

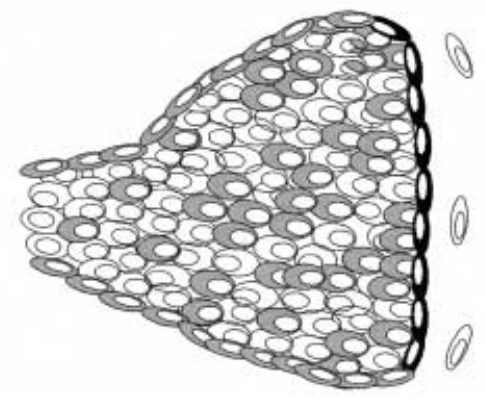

E18.5

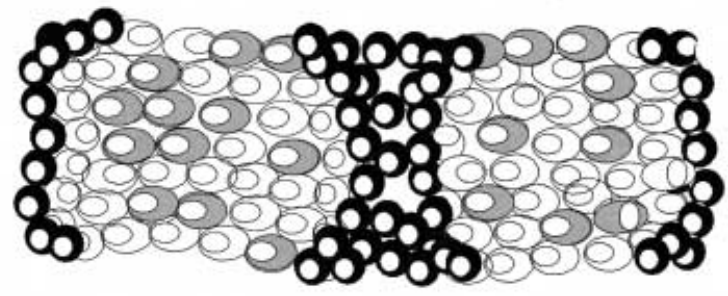

1

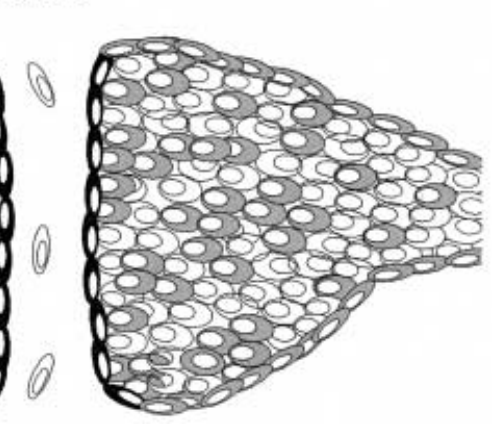

Figure 2 (see legend on page 245) 


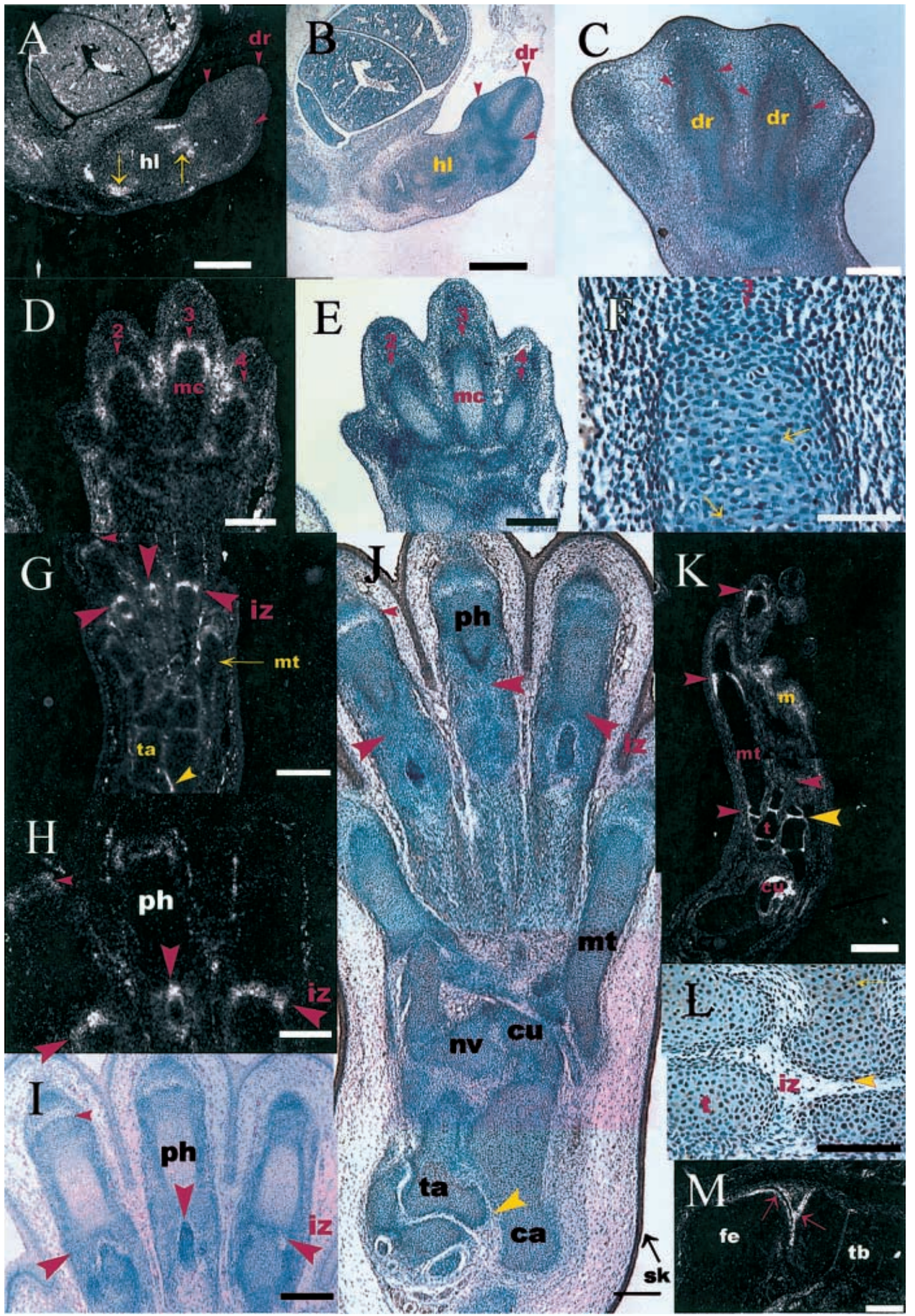

Figure 3 (see legend on page 246) 

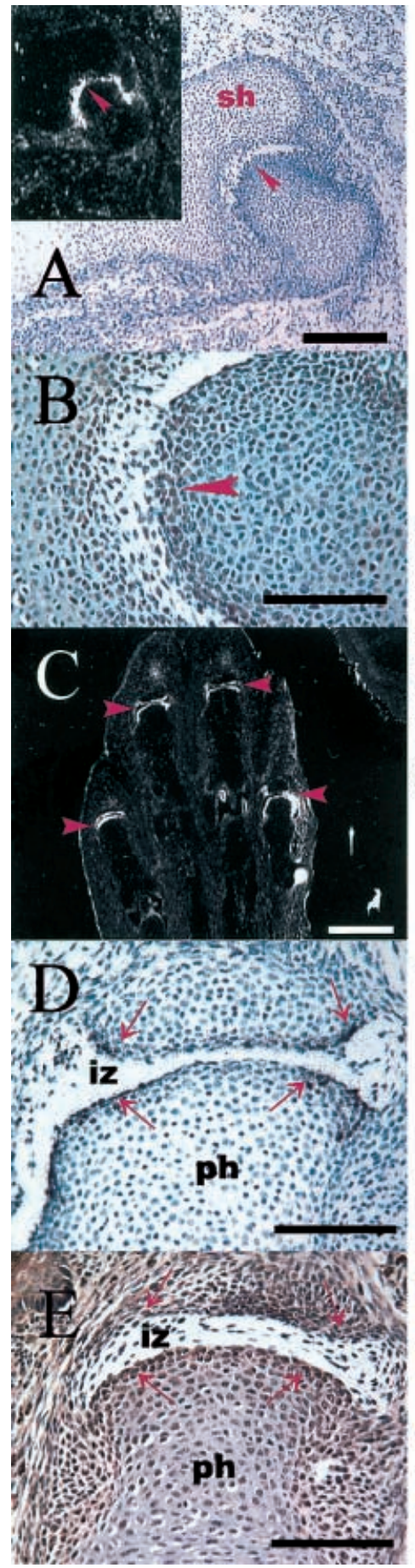
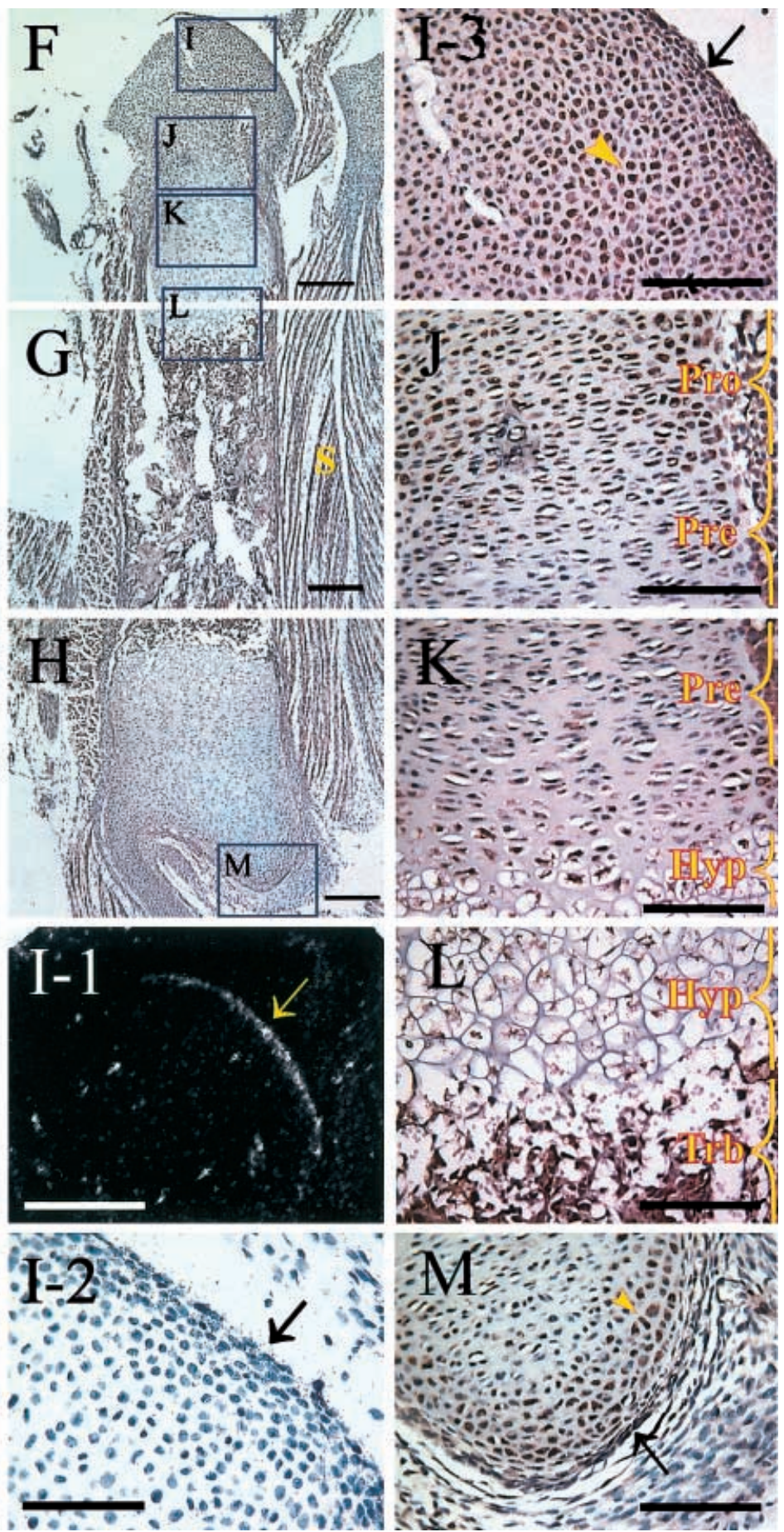

Figure 4 (see legend on page 246)
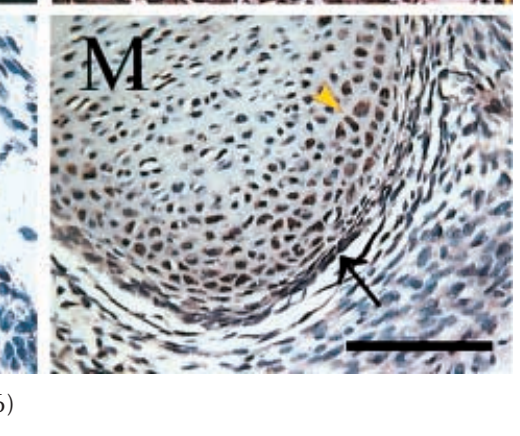
been accelerated by excess STC. This notion is also supported by a recent study showing that STC actively promotes bone nodule formation in rat fetal calvaria (Yoshiko et al. 2000). The present findings and those published recently on the distribution of STCir in mouse axial skeleton (Jiang et al. 2000) suggest that these effects probably occurred in part through the chondrocytes, given the high levels of STCir found at different stages of chondrocyte differentiation. Parathyroid hormone-related protein $(\mathrm{PTHrP})$ is just as widely distributed during embryogenesis and, interestingly, null mutants have profound defects in axial and appendicular skeleton as a result of accelerated endochondral mineralization. Because PTHrP is normally expressed in resting chondrocytes and targeted to slow the growth rate of prehypertrophic chondrocytes, the cells multiply and mineralize more rapidly in null mutants (Karaplis \& Kronenberg 1996, Lanske et al. 1996, Vortkamp et al. 1996). TGFs $\beta 1-3$, which are also expressed in perichondrocytes (Heine et al. 1987, Pelton et al. 1990), also appear to act as negative regulators of chondrocyte differentiation, but upstream of PTHrP (Serra et al. 1999). The data on STC collectively suggest that it, too, may function as a regulator of chondrogenesis.

\section{STC in the appendicular skeleton}

Over the early stages of appendicular skeletal development and before the onset of chondrogenesis, STC mRNA and protein co-localized in the general contours outlining future limbs (precartilaginous condensations) and were especially noticeable in more proximal areas where joint formation was about to take place. Its presence in such a spatially restricted manner, before overt cartilage development, is suggestive of a role in defining inceptive boundaries. Similarly, the distinctive patterns of expression of the gene in all joint interzone regions up to and including the time of cavitation, when it became confined to the articular surfaces, is equally suggestive of a role for STC in joint formation.

The patterns of STC gene expression and protein accumulation in appendicular skeletal joints, as summarized in Fig. 2, are highly unique relative to what has previously been described in the developing skeleton. Various proteins are found in developing cartilage and nearby structures during joint formation, including tenacin-C, syndecan-3 (Koyama et al. 1995), types I, II, and XI collagens, and keratan sulfate (Craig et al. 1987,

\begin{abstract}
Figure 1 STC mRNA and protein in developmental stages E11.5-E14.5 axial skeleton. (A) Darkfield ISH of E11.5 developing axial skeleton (sagittal view). Yellow arrow denotes gene expression in one sclerotome segment. (B) Higher power bright field ISH of sclerotome segments highlighted in panel A. Gene expression is high in each of the low-cell-density rostral zones (ro) and less evident in the higher-density caudal zones (ca). (C) Section closely adjacent to panel A, after ICC staining. STCir is evident in both the rostral and caudal zones of each sclerotomal condensation. Notice also the high STCir in the dorsal root ganglion (drg). (D) Darkfield ISH of E13.5 thoracic-lumbar-sacral vertebral region (sagittal view). Yellow arrows denote intense hybridization in sacral intervertebral discs (id). White arrowhead denotes lack of gene expression in thoracic intervertebral discs (th). The inset is a higher power ICC of the upper thoracic region (th). Yellow arrowheads in inset denote STCir perichondrium and chondrocytes of thoracic vertebral cartilage elements. STCir is also high in intervertebral disc cells (asterisk). c, cartilage primordium; ki; kidney. (E,F) Higher power ISH (E) and adjacent ICC (F) of sacral region in panel D. Arrows show co-localization of mRNA and protein to cells of the intervertebral disc regions (id) between developing vertebral bodies (vb). (G) Higher magnification of intervertebral disc (id) region in F. At this stage most STCir is confined to id cells expressing the gene, but is also evident in pre-chondrocytes of the vertebral bodies (vb). $(\mathrm{H})$ Darkfield ISH of E15.5 cervical vertebral elements (sagittal view). Red arrows denote low levels of gene expression in cells bordering the cartilage primordium of the basioccipital bone (bo), atlas C1 vertebra (C1) and axis C2 vertebra (C2); mu, muscle. (I) Adjacent ICC of E15.5 cervical vertebral elements in panel H. Red arrows show co-localization of STC protein to cells bordering the vertebral elements and cells within the cartilage primordium of the vertebrae. Abbreviations are as in panel H. (J) Higher power of C2 cartilage primordium in panel I. Red arrowheads denote high STCir in chondrocytes with dispersed chromatin (more active cells). Yellow arrows denote lack of STCir in those with condensed chromatin (less active cells). Scale bars represent $500 \mu \mathrm{m}$ in panels A and C, $200 \mu \mathrm{m}$ in B, D, H and I, $100 \mu \mathrm{m}$ in D, G and J, and $50 \mu \mathrm{m}$ in E and F.
\end{abstract}

Figure 2 Major patterns of STC mRNA and protein localization during axial and appendicular skeletal development. Black cells express the STC gene and exhibit STC immunoreactivity (STCir); gray cells do not appear to express the gene but nonetheless exhibit STCir, whereas white cells contain no STC mRNA or protein. Axial: Segmentation in vertebrates occurs with the condensation of mesoderm to form somites; over developmental stages E11-E13 in mice, these differentiate into dermatome, myotome and sclerotome, generating dermal, muscle and skeletal tissue respectively. Of interest here is the sclerotomal component giving rise to the axial skeleton. The top panel is representative of the E11.5 cervical-thoracic region as seen in Fig $1 \mathrm{~A}-\mathrm{C}$, where each sclerotome unit consists of a rostral zone of low cell density and a more dense caudal zone. Cells in the rostral zone produce STC and target it to cells in the caudal zone. The middle panel is representative of E14.5 axial skeleton after vertebral condensation. The gene is now expressed in the intervertebral disc primordium and targeted to precartilaginous cells within the vertebral bodies. Note that gene activity is restricted to the outermost layers of disc cells (as also seen in Fig. 1D,E). The bottom panel is of E18.5 axial skeleton as in Fig. $1 \mathrm{H}$; by this time the gene is uniquely expressed in perichondrial cells lining the vertebral bodies, from whence it targets underlying vertebral chondrocytes. Appendicular: Limb development begins with the condensation of precartilaginous cells in the limb buds. The top right panel is representative of E13.5 limb where cells have begun condensing into skeletal elements as in Fig. 2A-C. STC production is restricted to mesenchyme cells defining the longitudinal boundaries of each element and targeted to the condensing cells. Middle panel: in preparation for joint formation, cells defining the new joint margins express the STC gene at high levels and target chondrocytes in adjacent skeletal elements, as in Fig. 2G-J, K-L. By stage E18.5 (bottom) and following joint cavitation, STC gene expression becomes restricted to perichondrocytes on articular surfaces and targeted to underlying chondrocytes, as in Fig. $4 \mathrm{~A}-\mathrm{C}$ and $\mathrm{E}$. 
Nalin et al. 1995), in addition to hyaluronan and hyaluronan binding proteins (Pitsillides et al. 1995, Dowthwaite et al. 1998). However, surprisingly few genes have a pattern of expression comparable to that of STC in the developing joint itself. Moreover, although some genes are expressed in the joint region in a stage-specific manner (Gli3, Egr1, Bmp2, chordin), only growth and differentiation factor 5 (GDF5), shows significant overlap with STC mRNA at several stages of joint formation in mice. Like STC, GDF5 is expressed at high levels in the interzone regions of developing joints and around the periphery of long bones in the early stages of condensation. GDF5 has also been directly implicated in both joint formation and chondrogenesis, as GDF5 gene mutations are responsible for the skeletal abnormalities in brachypodism. In these mice, both long bone length and phalanx number are decreased, presumably as a result of regional failure to cleave the early condensations into two separate parts (Storm et al. 1994, Storm \& Kingsley 1996). GDF5 mutations also cause various skeletal dysplasias in humans (Storm \& Kingsley 1999). The GDF5 receptor, Alk-6, is expressed in epiphyseal chondrocytes next to the joint (Dewulf et al. 1995, Zoo et al. 1997, Francis-West et al. 1999b); as shown in the present study, these chondrocytes contain/sequester large amounts of STC protein at specific developmental stages. Also, like STC, the GDF5 gene is expressed in both synovial and fibrocartilaginous joints (Storm et al. 1994). Therefore, given its similar distribution patterns, it is likely that STC is involved in early patterning, joint formation and chondrogenesis. In this regard, it is interesting to note that a recent review proposed the joint as a 'signaling center' from which numerous molecules signal in paracrine fashion to control skeletogenesis in the juxtaposed cartilage elements (Francis-West et al. 1999a). In this context, STC appears to have the necessary characteristics of a 'signaling center' molecule.

Finally, it is interesting to note that, in bone, STC was produced in mesenchyme cells and sequestered by chondrocytes, much as in kidney and testis, where it is produced in mesenchyme and sequestered by epithelial cells (Stasko \& Wagner 2001); the common denominator is that the protein is produced in less differentiated cells

Figure 3 STC mRNA and protein in developmental stages E12.5 and E16.5 appendicular skeleton. (A) Low power darkfield ISH of stage E12.5 hind limb (sagittal view). Yellow arrows denote areas of high STC gene expression proximal and distal to developing hind limb elements (hl). Red arrowheads denote lower levels of gene expression in regions between digital rays (dr). (B) Brightfield ISH of hind limb (hl) in panel A. Red arrowheads denote digital rays (dr). (C) ICC of E12.5 hind limb. Red arrowheads denote STCir in cells surrounding digital rays (dr). (D) Darkfield and (E) brightfield ISH of E13.5 fore limb (coronal view). Red arrowheads denote gene expression in the most distal mesenchymal cell condensations of the 2nd, 3rd and 4th metacarpal elements (mc). (F) Adjacent high power ICC of 3rd metacarpal $(\mathrm{mc})$ shown in panel E. Yellow arrows denote light STCir in select chondrocytes of the third metacarpal. (G) Low power darkfield ISH of E15.5 hind limb (coronal view). Large red arrowheads denote high levels of STC mRNA in metatarsal-phalangeal interzone (iz) regions of $2 \mathrm{nd}$, 3rd and 4th digits. Small red arrowheads denote mRNA at the distal end of a phalanx in putative interphalangeal joint interzone. Yellow arrowhead denotes mRNA in cavitating interzone between the talus (ta) and calcaneus tarsal elements. The yellow arrow denotes a metatarsal $(\mathrm{mt})$. (H) Higher power darkfield ISH of metatarsal-phalangeal (ph) joint interzone region in panel G. (I) Brightfield ISH of panel $\mathrm{H}$. Red arrowheads denote metatarsal-phalangeal (ph) and interphalangeal joint regions where gene expression is greatest. (J) Near adjacent ICC of E15.5 hind limb in panels G-I (coronal view). Arrowheads correspond to those shown in panels G-I. STCir is greatest in skin (sk), followed by chondrocytes in cartilaginous elements, and is least in regions expressing perichondrial and interzone cells. The STCir in skin is probably derived from several sources. ca, calcaneus; cu, cuboideum; mt, metatarsal; nv, naviculare; ph, phalanx; ta, talus. (K) Low power darkfield ISH of tarsal (t) and metatarsal elements (mt) in E16.5 hind limb (coronal view). Arrowheads denote gene expression restricted to interzone regions. Moderate gene activity is also evident in the ventral mesenchyme $(\mathrm{m})$ of the hind limb. $\mathrm{cu}$, cuboideum. (L) Higher power ICC of tarsal elements (t) and interzone region denoted by the yellow arrowhead in panel K. Note the weak STCir in gene-expressing interzone cells (iz) and perichondrocytes (yellow arrowhead); most STCir is instead confined to the underlying chondrocytes of cartilage elements (yellow arrow). (M) Darkfield ISH of E15.5 knee-joint region (sagittal view). Red arrows denote STC mRNA in cavitating knee-joint region between femur (fe) and tibia (tb). Scale bars represent $500 \mu \mathrm{m}$ in $\mathrm{A}, \mathrm{B}, \mathrm{G}$ and $\mathrm{K}, 200 \mu \mathrm{m}$ in $\mathrm{C}-\mathrm{E}, \mathrm{H}-\mathrm{J}$ and $\mathrm{M}$, and $100 \mu \mathrm{m}$ in $\mathrm{F}$ and $\mathrm{L}$.

Figure 4 STC mRNA and protein in later stages of appendicular skeleton. (A) STC gene expression in developmental stage E14.5 shoulder (sh) joint (sagittal view). The inset shows darkfield ISH of the shoulder joint in panel A and the high level of expression of the gene in the interzone region (red arrowhead). (B) ICC staining adjacent to panel A, showing STCir in gene-expressing interzone cells (red arrowhead) and some underlying hyaline chondrocytes. (C) Low power darkfield ISH of E18.5 forelimb digits (coronal view). Red arrowheads denote specific hybridization to cavitating interphalangeal joint regions. (D) High power brightfield ISH of 2nd interphalangeal cavitating interzone (iz) in panel C (coronal view). Lower red arrows highlight black silver grains on the distal articular surfaces of the proximal phalanx (ph), indicative of STC gene expression. The same pattern is also apparent on the opposing proximal edge of the more distal (medial) phalanx (upper red arrows). (E) ICC adjacent to panel D. Red arrows denote STCir in articular cartilage cells and underlying hyaline cartilage cells on proximal and distal articular surfaces. $(\mathrm{F}-\mathrm{H})$ Low power composite image of an E17.5 femur (sagittal view). Boxed areas in the composite are shown at higher power in panels $\mathrm{I}-\mathrm{M}$. Panels $\mathrm{J}-\mathrm{L}$ define the growth plate. $(\mathrm{I}-1, \mathrm{I}-2)$ Gene expression is restricted to perichondrocytes on articular surfaces, as shown under darkfield illumination in panel I-1 and under brightfield in panel I-2 (arrows). (I-3) STCir co-localizes in cells expressing the gene (black arrow), but was also evident in chondrocytes of the underlying resting zone (yellow arrowhead). (J) STCir is also evident in growth plate chondrocytes of the proliferative (Pro) and prehypertrophic zones (Pre). (K) STCir is present in prehypertrophic and hypertrophic chondrocytes (Hyp) of the growth plate. (L) STCir is weakly evident in hypertrophic chondrocytes, but highly evident in osteoblasts of the trabecular zone (Trb). (M) Similar to the distal end (panel I-3), STCir was also evident in the perichondrocytes (black arrow) and underlying resting chondrocytes (yellow arrowhead) in the proximal femur. Scale bars represent $500 \mu \mathrm{m}$ in $\mathrm{C}, 200 \mu \mathrm{m}$ in $\mathrm{A}, \mathrm{F}-\mathrm{H}$ and $\mathrm{I}-1$, and $100 \mu \mathrm{m}$ in $\mathrm{B}, \mathrm{D}, \mathrm{E}, \mathrm{I}-2, \mathrm{I}-3$ and $\mathrm{J}-\mathrm{M}$. 
and targeted/sequestered by those undergoing active differentiation. This interpretation is, of course, based upon our aforementioned hypothesis that the cells containing only STC protein do not in fact express the gene. However, if the sequestering hypothesis proves to be true, then its significance in terms of development and cell signaling generally are questions that will need to be addressed. This is especially true in the case of tissues such as skin, that contain by far the highest levels of STCir but no evidence of gene activity. In this regard, one compartment that has not yet been mentioned and yet should be considered as a source of STC for cells with no apparent gene activity (chrondrocytes and keratinocytes) is maternal serum, which contains high levels of STCir during the latter half of gestation (Deol et al. 2000). It should also prove interesting to see if $S T C$ expression persists with the subsequent maturation and refinement of the articular cartilage joint surface in the postnatal period, and which, if any, patterns of expression are maintained throughout the adult life span. A recent report has revealed that STC mRNA is present in adult mouse chondrocytes and osteoblasts using digoxigenin-based in situ hybridization and northern blot analysis (Yoshiko et al. 1999). Although relative levels of expression and putative targets were not investigated, these findings nonetheless imply that STC continues to be of importance to bone biology long after skeleton maturation. In support of these findings, we also observed high levels of STC protein in trabecular osteoblasts of the stage E17.5 femur. However, whereas we readily observed STC mRNA in articular perichondrocytes, we saw no evidence of gene activity in osteoblasts, using ${ }^{35}$ S-labeled riboprobes. The discrepancy in our findings may simply reflect the different in situ techniques used (isotopic and non-isotopic in situ hybridizations). Therefore, if osteoblast-like cells do express the STC gene, it is probably in concentrations that are undetectable with ${ }^{35} \mathrm{~S}$-labeled riboprobes.

In summary, therefore, STC was transiently expressed in a rostral-caudal fashion in intervertebral disc mesenchyme cells during vertebral development, and targeted to vertebral chondrocytes. The pattern of expression in appendicular skeleton was equally striking. Early on, STC gene expression defined the initial lengths of bone primordia and the gene product was targeted to precartilaginous condensations of mesenchyme cells. Later on, STC was highly expressed in interzone cells defining future joints and in perichondrocytes lining the joints after cavitation. Underlying resting, proliferating and hypertrophic chondrocytes appeared to be the targets of STC both during and after joint formation. Therefore, as in urogenital development, STC was made in undifferentiated mesenchyme cells and sequestered by differentiating cells. In this instance, however, the patterns of distribution of mRNA and protein were indicative of a role in early skeletal patterning and joint formation. It may be hoped that future studies will delineate both the interactions of
STC with other bone morphogens and its precise roles in skeletal development.

\section{Acknowledgements}

Supported by an operating grant from the Medical Research Council of Canada to G F Wagner (MT-11738). We thank Dr Michael Underhill for helpful comments on the manuscript and Dr Gerry Kidder for technical assistance. We also acknowledge the assistance of Human Genome Sciences Ltd (Rockville, MD, USA) in providing the recombinant human STC used in antibody production and immunocytochemical blocking studies.

\section{References}

Chang ACM, Janosi J, Hulsbeek M, DeJong D, Jeffery KL, Noble JR \& Reddel RR 1995 A novel human cDNA highly homologous to the fish hormone stanniocalcin. Molecular and Cellular Endocrinology 112 241-247.

Chang ACM, Dunham MA, Jeffery KL \& Reddel RR 1996 Molecular cloning and characterization of mouse stanniocalcin cDNA. Molecular and Cellular Endocrinology 124 185-187.

Craig FM, Bently G \& Archer CW 1987 The spatial and temporal pattern of collagens I and II and keratan sulphate in the developing chick metatarsophalangeal joint. Development 99 383-391.

Deol HK, Varghese R, Wagner GF \& DiMattia GE 2000 Dynamic regulation of mouse ovarian stanniocalcin expression during gestation and lactation. Endocrinology 141 3412-3412.

Deutsch U, Dressler GR \& Gruss P 1988 Pax 1, a member of a paired box homologous murine gene family, is expressed in segmented structures during development. Cell $\mathbf{5 3}$ 617-625.

Dewulf N, Verschueren K, Lonnoy O, Moren A, Grimsby S, Spiegle KV, Miyazono K, Huylebroeck D \& Dijke PT 1995 Distinct spatial and temporal expression patterns of two type I receptors for bone morphogenetic proteins during mouse embryogenesis. Endocrinology 136 2652-2663.

Dowthwaite GP, Edwards JCW \& Pitsillides AA 1998 An essential role for the interaction between hyaluronan and hyaluronan binding proteins during joint development. Journal of Histochemistry and Cytochemistry 46 641-651.

Feijen A, Goumans MJ \& van den Eijnden-van raaij AJM 1994 Expression of activin subunits, activin receptors and follistatin in postimplantation mouse embryos suggests specific developmental functions for different activins. Development 120 3621-3637.

Francis-West PH, Aldelfattah A, Chen P, Allen C, Parish J, Ladher R, Allen S, MacPherson S, Luyten FP \& Archer CW 1999a Mechanisms of GDF-5 action during skeletal development. Development 126 1305-1315.

Francis-West PH, Parish J, Lee K \& Archer CW 1999b BMP/ GDF-signalling interactions during synovial joint development. Cell and Tissue Research 296 111-119.

Heine HI, Munoz EF, Flanders KC, Ellingsworth LR, Lam H-YP, Thompson NL, Roberts AB \& Sporn MB 1987 Role of transforming growth factor- $\beta$ in the development of the mouse embryo. Journal of Cell Biology 105 2861-2876.

Jiang WQ, Chang AC, Satoh M, Furuichi Y, Tam PP \& Reddel RR 2000 The distribution of stanniocalcin 1 protein in fetal mouse tissues suggests a role in bone and muscle development. Journal of Endocrinology 165 457-66.

Karaplis AC \& Kronenberg HM 1996 Physiological roles for parathyroid hormone-related protein: lessons from gene knockout mice. Vitamins and Hormones 52 177-193. 
Koyama E, Leatherman JL, Shimazu A, Nah H-D \& Pacifici M 1995 Syndecan-3, tenascin-C, and the development of cartilaginous skeletal elements and joints in the chick limbs. Developmental Dynamics 203 152-162.

Lanske B, Karapilis AC, Lee K, Luz A, Vortkamp A, Pirro A, Karperien M, Defize LHK, Ho C, Mulligan RC, Abou-Samra A-B, Juppner H, Segre GV \& Kronenberg HM 1996 PTH/PTHrP receptor in early development and indian hedgehog-regulated bone growth. Science 273 663-666.

McCudden C, Kogon M, DiMattia GE \& Wagner GF 2001 Novel expression of the stanniocalcin gene in fish. Journal of Endocrinology $17133-44$.

Madsen KL, Tavernini MM, Yachimec C, Mendrick DL, Alfonso PJ, Buergin M, Olsen HS, Antonaccio MJ, Thompson AB \& Fedorak RN 1998 Stanniocalcin: a novel protein regulating calcium and phosphate transport across mammalian intestine. American Journal of Physiology 274 G96-G102.

Millan FA, Denhez F, Kondaiah P \& Akhurst RJ 1991 Embryonic gene expression patterns of TGF $\beta 1, \beta 2$ and $\beta 3$ suggest different developmental functions in vivo. Development 111 131-144.

Nalin AM, Greenlee TK \& Sandell LJ 1995 Collagen gene expression during development of avian synovial joints: transient expression of types II and XI collagen genes in the joint capsule. Developmental Dynamics 203 352-362.

Niu PD, Radman DP, Jaworski EM, Deol H, Gentz R, Su J, Olsen HS \& Wagner GF 2000 Development of a human stanniocalcin radioimmunoassay: serum and tissue hormone levels and pharmacokinetics in the rat. Molecular and Cellular Endocrinology 162 131-144.

Oliver G, Wright CVE, Hardwicke J \& Robertis EMDe 1988 Differential antero-posterior expression of two proteins encoded by a homeobox gene in Xenopus and mouse embryos EMBO Journal 7 3199-3209.

Olsen HS, Cepeda MA, Zhang QQ, Rosen CA, Vozzolo BL \& Wagner GF 1996 Human stanniocalcin: a possible hormonal regulator of mineral metabolism. PNAS 132 903-909.

Pelton RW, Dickinson ME, Moses HL \& Hogan BLM 1990 In situ hybridization analysis of TGF $\beta 3$ RNA expression during mouse development: comparative studies with TGF $\beta 1$ and $\beta 2$. Development 110 609-620.

Pitsillides AA, Archer CW, Prehm P, Bayliss MT \& Edwards JCW 1995 Alterations in hyaluronan synthesis during developing joint cavitation. Journal of Histochemistry and Cytochemistry 43 263-273.

Schmid P, Cox D, Bilbe G, Maier R \& McMaster GK 1991 Differential expression of TGF $\beta 1, \beta 2$ and $\beta 3$ genes during mouse embryogenesis. Development 111 117-130.

Serra R, Karaplis A \& Sohn P 1999 Parathyroid hormone-related peptide (PTHrP)-dependent and -independent effects of transforming growth factor $\beta$ (TGF $\beta$ ) on endochondral bone formation. Journal of Cell Biology 145 783-794.

Stasko SE \& Wagner GF 2001 Stanniocalcin gene expression during mouse urogenital development: a possible role in mesenchymal-epithelial signaling. Developmental Dynamics 220 49-59.

Stasko SE, DiMattia GE \& Wagner GF 2001 Dynamic changes in stanniocalcin gene expression in the mouse uterus during early implantation. Molecular and Cellular Endocrinology 174 145-150.
Storm EE \& Kingsley DM 1996 Joint patterning defects caused by single and double mutations in members of the bone morphogenic protein (BMP) family. Development 122 3969-3979.

Storm EE \& Kingsley DM 1999 GDF5 coordinates bone development and joint formation during digit development. Developmental Biology $20911-27$.

Storm EE, Huynh TV, Copeland NG, Jenkins NA, Kingsley DM \& Lee S-E 1994 Limb alterations in brachypodism mice due to mutations in a new member of the TGF $\beta$-superfamily. Nature 368 639-643.

Varghese R, Wong CKC, Deol H, Wagner GF \& DiMattia GE 1998 Comparative analysis of mammalian stanniocalcin genes. Endocrinology 139 4714-4725.

Varghese RP, Bialek S-P, Yee GF, Wagner GE \& DiMattia 2000 Over-expression of stanniocalcin in the mouse affects growth and reproductive potential. Proceedings of The Endocrine Society, 82nd Annual Meeting, Toronto, Ontario.

Vortkamp A, Lee K, Lanske B, Segre GV, Kronenberg HM \& Tabin CJ 1996 Regulation of rate of cartilage differentiation by indian hedgehog and PTH-related protein. Science 273 613-622.

Wagner GF 1994 The molecular biology of the corpuscles of stannius and regulation of stanniocalcin gene expression. In Fish Physiology, vol 13, ch 9, pp 273-306. Eds N Sherwood \& C Hew. New York: Academic Press.

Wagner GF, Hampong M, Park CM, Miliken C, Copp DH \& Freisen FG 1986 Purification, characterization and bioassay of teleocalcin (stanniocalcin), a glycoprotein from corpuscles of Stannius. General and Comparative Endocrinology 63 481-491.

Wagner GF, Vozzolo BL, Jaworski E, Haddad M, Kline RL, Olsen HS, Rosen CA, Davidson MB \& Renfro JL 1997 Human stanniocalcin inhibits renal phosphate excretion in the rat. Bone and Mineral Research 12 165-171.

Wallin J, Wilting J, Koseki H, Fritsch R, Christ B \& Balling R 1994 The role of Pax-1 in axail skeleton development. Development 120 1109-1121.

Yoshiko Y, Son A, Maeda S, Igarashi A, Takano S, Hu J \& Maeda N 1999 Evidence for stanniocalcin gene expression in mammalian bone. Endocrinology 140 1869-1874.

Yoshiko Y, Maeda S \& Aubin JE 2000 Stanniocalcin is a novel autocrine/paracroine factor necessary for bone formation. Journal of Bone and Mineral Research 15 (Suppl 1) Abstract M194.

Zhang KZ, Westberg JA, Paetau A, von Boguslawsky K, Lindsberg P, Erlander M, Guo H, Su J, Olsen HS \& Andersson LC 1998 High expression of stanniocalcin in differentiated brain neurons. American Journal of Pathology 153 439-445.

Zhang KZ, Lindsberg PJ, Tatlisumak T, Kaste M, Olsen HS \& Andersson LC 2000 Stanniocalcin: a molecular guard of neurons during cerebral ischemia. PNAS 97 3637-3642.

Zoo H, Wieser R, Massague J \& Niswander L 1997 Distinct roles of type I bone morphogenetic protein receptors in the formation and differentiation of cartilage. Genes and Development 11 2191-2203.

Received 27 April 2001

Accepted 20 July 2001 